\title{
DYNAMICAL SYSTEMS WHOSE ORBIT SPACES ARE NEARLY HAUSDORFF
}

\author{
ROGER C. MCCANN
}

\begin{abstract}
Consider a continuous flow on a locally compact, separable, metric space. If the set of nonperiodic recurrent points is nowhere dense, then there is an open, dense, invariant subset of the phase space which has a Hausdorff orbit space. A separatrix is defined to be a trajectory which is in the closure of the set of trajectories at which the orbit space is not Hausdorff. If the flow is completely unstable, then the set of points which lie on separatrices is nowhere dense in the phase space.
\end{abstract}

Introduction. Orbit spaces and their topological properties arise in a natural way when the trajectorial structure of a continuous flow is studied, e.g., in [2], [4], and [6]. One of the fundamental concepts of trajectorial structure is that of a parallelizable flow. Parallelizable flows have been studied and characterized in [1], [2], and [7, \$2.4]. If the flow is parallelizable, then the natural mapping of the phase space onto the orbit space is the projection of a fiber bundle. One immediate consequence of parallelizability is that the orbit space is Hausdorff. If a flow on a locally compact, separable, metric space has a Hausdorff orbit space, then there is a natural generalization of the fiber bundle structure for parallelizable flows [6]. Hence, flows with Hausdorff orbit spaces are a natural generalization of flows which are parallelizable. Flows with Hausdorff orbit spaces also arise in the study of completely unstable flows [4]. Clearly, it is very restive to assume that a flow has a Hausdorff orbit space. In this paper we will show that if a flow on a locally compact, separable, metric space has a nowhere dense set of nonperiodic recurrent points, then there is an open, dense, invariant subset $U$ of the phase space such that the orbit space with respect to $U$ is Hausdorff. A separatrix is a trajectory which is in the closure of the set of trajectories at which the orbit space is not Hausdorff. In the case that the flow is completely unstable, we will show that the set of points which lie on separatrices is nowhere dense in the phase-space.

1. Basic definitions and properties of continuous flow. Throughout this paper $X$ will be a locally compact, separable, metric space and $R, R^{+}$will denote the reals and nonnegative reals, respectively.

A continuous flow $\pi$ on $X$ is a continuous mapping of $X \times R$ onto $X$

Received by the editors September 4, 1978.

AMS (MOS) subject classifications (1970). Primary 34C35; Secondary 54H20.

Key words and phrases. Dynamical systems, orbit spaces, separatrix. 
satisfying the following axioms (where $x \pi t=\pi(x, t)$ for $(x, t) \in X \times R)$ :

(1) $x \pi 0=x$ for $x \in X$,

(2) $(x \pi t) \pi s=x \pi(t+s)$ for $x \in X$ and $t, x \in R$.

For $A \subset X$ and $B \subset R, A \pi B$ will denote the set $\{x \pi t: x \in A, t \in B\}$. In the special case $B=R$ we will write $C(A)$ instead of $A \pi R$. If $A$ consists of a single element $x$, we will write $x \pi B$ instead of $\{x\} \pi B$. For $x \in X, C(x)$ is called the trajectory through $x$. A set $A \subset X$ is said to be invariant if $C(A)=A$. If $C(x)=\{x\}, x$ is called a critical point. The set of all critical points will be denoted by $S$. If $x \in X$ is noncritical and $x \pi t=x$ for some $t \neq 0$, then $x$ is said to be periodic. The set of all periodic points will be denoted by $\mathscr{P}$. If $x \in \mathscr{P}$ and $X$ is Hausdorff, then $t_{x}=\inf \{t>0: x \pi t=x\}$ exists and is positive. The mapping defined by $x \rightarrow t_{x}$ is called the fundamental period function.

If $x, y \in X$, we write $x C y$ if and only if $x \in C(y)$. Evidently $C$ is an equivalence relation on $X$. The topology of $X / C$ will be the quotient topology. $X / C$ is called the orbit space of $\pi$. If $A \subset X$ is invariant we will write $A / C$ for the orbit space of $\left.\pi\right|_{(A \times R)}$.

Let $x \in X$. Then $\mathrm{L}^{+}(x)\left[L^{-}(x)\right]$ will denote the positive [negative] limit set of $x$. A flow is said to be unstable if no limit set is compact. An $x \in X$ is called recurrent if $\overline{C(x)}=L^{+}(x)=L^{-}(x)$. $\Re$ will denote the set of all recurrent points. An $x \in X$ is called wandering if there is a neighborhood $U$ of $x$ and an $s \in R$ such that $(U \pi t) \cap U=\varnothing$ for all $t>s$. $W$ will denote the set of all wandering points. Evidently $W$ is open. A flow is called completely unstable if every $x \in X$ is wandering, i.e., $W=X$.

The prolongation $D(x)$ of $x$ is $D(x)=D^{+}(x) \cup D^{-}(x)$, where $D^{+}(x)$ is the set $\left\{y \in X\right.$ : there exist sequences $\left\{x_{i}\right\}$ in $X$ and $\left\{t_{i}\right\}$ in $R^{+}$such that $x_{i} \rightarrow x$ and $\left.x_{i} \pi t_{i} \rightarrow y\right\}$ and $D^{-}(x)$ is defined analogously. It can be shown that $X / C$ is Hausdorff if and only if $C(x)=D(x)$ for every $x \in X$ (e.g., see [6, Proposition 2.4]).

The prolongational limit set $J(x)$ of $x$ is $J(x)=J^{+}(x) \cup J^{-}(x)$ where $J^{+}(x)$ is the set $\left\{y \in X\right.$ : there exist sequences $\left\{x_{i}\right\}$ in $X$ and $\left\{t_{i}\right\}$ in $R^{+}$such that $x_{i} \rightarrow x, t_{i} \rightarrow \infty$, and $\left.x_{i} \pi t_{i} \rightarrow y\right\}$ and $J^{-}(y)$ is defined analogously. $\pi$ is said to be parallelizable if there exist a subset $K$ of $X$ and a homeomorphism $h: X \rightarrow K \times R$ such that $K \pi R=X$ and $h(x \pi t)=(x, t)$ for every $(x, t) \in K$ $\times R$. If $X$ is a locally compact, separable, metric space, then $\pi$ is parallelizable if and only if $J(x)=\varnothing$ for each $x \in X$ [1, Theorem 3].

A subset $T$ of $X$ is called a local section of extent $\lambda, \lambda>0$, if $(T \pi t) \cap T=$ $\varnothing$ for $0<|t|<\lambda$. $T$ is said to generate neighborhoods of $x \in T$ if for arbitrarily small $a>0, T \pi(-a, a)$ is a neighborhood of $x . T$ is said to be interior in $X$ if, for sufficiently small $a>0, T \pi(-a, a)$ is an open subset of $X$. If $x \in X$ is a noncritical point of $\pi$ and $X$ is a Tychonoff space, then there exists a local section $T$ which generates neighborhoods of $x[2, \S \mathrm{VI}$, Theorem 2.12]. It can be shown that $T$ may be chosen to be interior in $X$. If $T$ is an 
interior local section and $U$ an open set, then $T \cap U$ is an interior local section.

If $U$ is a subset of $X$, then int $U$ and $\partial U$ will denote the interior and the boundary of $U$ respectively.

\section{Orbit spaces.}

Proposition 2.1. If $W \neq \varnothing$, then there is an open, dense, invariant subset $W_{1}$ of $W$ such that $J\left(W_{1}\right) \cap W_{1}=\varnothing$.

Proof. Evidently $x \in W$ if and only if there is an invariant neighborhood $U$ of $x$ such that $J(U) \cap U=\varnothing$. Set $F=\{U \subset W: U$ open and invariant, $J(U) \cap U=\varnothing\}$. Let $\left\{U_{i}\right\}$ be a chain in $F$ and set $V=\cup U_{i}$. Since $J\left(U_{i}\right) \cap$ $U_{i}=\varnothing$ for each $i$, it is easy to verify that $J(V) \cap V=\varnothing$. Hence, $V$ is a maximal element for $\left\{U_{i}\right\}$. By Zorn's lemma there is a maximal element $W_{1}$ of $F$. Suppose $W_{1}$ is not dense in $W$. Then there exist an $x \in W-W_{1}$ and an open invariant neighborhood $U_{0} \subset W-\bar{W}_{1}$ of $x$ such that $J\left(U_{0}\right) \cap U_{0}=$ $\varnothing$. Then $U_{0} \cup W_{1} \in F$. This contradicts the maximality of $W_{1}$. Hence, $W_{1}$ is dense in $W$.

Proposition 2.2. If int $\mathscr{P} \neq \varnothing$, then the set $\mathcal{P}_{1}$ of points at which the fundamental period function is continuous is an open, dense subset of int $\mathscr{P}$.

Proof. Let $\Upsilon(x)$ denote the fundamental period of $x \in \mathscr{P}$. It is known and easy to prove that $\lim \inf _{y \rightarrow x} \Upsilon(y) \geqslant \Upsilon(x)$, i.e., $\Upsilon$ is lower semicontinuous. Theorem $1[3$, p. 394] states: "The set $D$ of points of discontinuity of a $B$ measurable function of class 1 is of the first category". Hence, $\Upsilon$ is continuous on a dense subset of $\mathcal{P}$. Let $\Upsilon$ be continuous at $x \in$ int $\mathcal{P}$ and let $T$ be an interior local section of extent $\lambda$ which generates neighborhoods of $x$ and is such that $\bar{T} \cap C(x)=\{x\}$. We will first show that there is an open neighborhood $U$ of $x$ such that $C(y) \cap T=\{y\}$ for every $y \in U \cap T$. Suppose the contrary. Then there exists a sequence $\left\{x_{i}\right\}$ in $\mathscr{P} \cap T$ such that $x_{i} \rightarrow x$ and $x_{i} \pi t_{i} \in T$ for some $t_{i} \in\left(0, \Upsilon\left(x_{i}\right)\right)$. Since $y \pi(\Upsilon(y)-a)=y \pi(-a)$ for every $a \in R$ and $T$ has extent $\lambda$, eventually we must have

$$
t_{i} \in\left[\lambda, \Upsilon\left(x_{i}\right)-\lambda\right] \subset\left[\lambda, \Upsilon(x)-\frac{1}{2} \lambda\right] \text {. }
$$

Let $\left\{s_{i}\right\}$ be any convergent subsequence of $\left\{t_{i}\right\}$ with $s_{i} \rightarrow s \in\left[\lambda, \Upsilon(x)-\frac{1}{2} \lambda\right]$. Then $x \pi s \leftarrow x_{i} \pi s_{i} \in T$. This contradicts the assumption that $\bar{T} \cap C(x)=$ $\{x\}$. Hence, there is a neighborhood $U$ of $x$ such that $C(y) \cap T=\{y\}$ for every $y \in T \cap U$. Without loss of generality we may assume that $T$ was chosen originally so that $T \subset U \cap$ (int $\mathscr{P}$ ). We will now show that $\Upsilon$ is continuous at each point of $T$. Let $y \in T$ and $\left\{y_{i}\right\}$ be a sequence in $X$ such that $y_{i} \rightarrow y$. Since $T$ is interior and $T \subset$ int $\mathscr{P}$ we may assume that $y_{i} \in C(T)$ $\cap \mathcal{P}$ for each $i$. For each $a \in\left(0, \frac{1}{2} \Upsilon(y)\right), T \pi(-a, a)$ is a neighborhood of $y=y \pi \Upsilon(y)$. Eventually $T \pi(-a, a)$ is a neighborhood of $y_{i}$ and $y_{i} \pi \Upsilon(y)$. Then there exist $s_{i}, t_{i} \in(-a, a)$ such that $y_{i} \pi s_{i} \in T$ and $\left(y_{i} \pi \Upsilon(y)\right) \pi t_{i} \in T$. Since 


$$
\left(y_{i} \pi \Upsilon(y)\right) \pi t_{i}=\left(y_{i} \pi s_{i}\right) \pi\left(\Upsilon(y)+t_{i}-s_{i}\right)
$$

and

$$
C\left(y_{i} \pi s_{i}\right) \cap T=\left\{y_{i} \pi s_{i}\right\}
$$

we must have $\Upsilon\left(y_{i}\right)$ is a positive integer multiple of $\Upsilon(y)+t_{i}-s_{i}$. Hence, eventually $\Upsilon\left(y_{i}\right) \leqslant \Upsilon(y)+2 a$. Since $a \in\left(0, \frac{1}{2} \Upsilon(y)\right)$ was arbitrary we have lim $\sup _{z \rightarrow y} \Upsilon(z) \leqslant \Upsilon(y)$. This coupled with the lower semicontinuity of $\Upsilon$ yields the continuity of $\Upsilon$ on $T$ and, hence, on $C(T)$ which is an open neighborhood of $x$. The desired result follows.

Proposition 2.3. Let $W_{1}$ and $\mathcal{P}_{1}$ be as in Propositions 1 and 2. Set $\delta_{1}=$ int $\delta$. Then each of $W_{1}, \mathcal{P}_{1}$, and $\S_{1}$ has a Hausdorff orbit space.

Proof. Since $J\left(W_{1}\right) \cap W_{1}=\varnothing$, the flow $\left.\pi\right|_{W_{1}}$ is parrallelizable. It follows directly that $W_{1} / C$ is Hausdorff. Since $\Upsilon$ is continuous on $\mathscr{P}_{1}$ it is easy to show that $C(x)=D(x)$ for every $x \in \mathscr{P}_{1}$. Hence, $\mathscr{P}_{1} / C$ is Hausdorff. Likewise it is easy to show that $C(x)=D(x)$ for every $x \in \delta_{1}$ so that $\delta_{1} / C$ is Hausdorff.

THEOREM 2.4. Let $\pi$ be a continuous flow on a locally compact, separable, metric space. Then there is an open, dense, invariant subset $U$ of $X-(\Re-$ (int $\mathcal{P} \cup$ int $\delta$ )) such that the orbit space of $U$ is Hausdorff.

Proof. In [5, p. 29] it is shown that $X=\overline{W \cup R}$. Let $W_{1}, \mathscr{P}_{1}$ and $\delta_{1}$ be as in Propositions 1-3. Then

$$
X=\bar{W} \cup \bar{\Re}=\bar{W}_{1} \cup \overline{\mathscr{P}}_{1} \cup \overline{\mathcal{S}}_{1} \cup \overline{\left(\Re-\left(\text { int } \mathscr{P}_{1} \cup \text { int } \mathcal{S}_{1}\right)\right)} .
$$

It follows directly that $U=W_{1} \cup \mathscr{P}_{1} \cup \delta_{1}$ is an open dense subset of $X-(\Re-($ int $\mathscr{P} \cup$ int $\delta))$. Since $W_{1}, \mathscr{P}_{1}, \delta_{1}$ are open, disjoint, invariant sets with Hausdorff orbit spaces, it follows that $U$ has a Hausdorff orbit space.

\section{Separatrices.}

Definition 3.1. Two trajectories $C_{1}$ and $C_{2}$ are said to be inseparable if any two neighborhoods of $C_{1}$ and $C_{2}$ in $X / C$ have nonempty intersection. A separatrix is a trajectory which lies in the closure of the inseparable elements of $X / C$.

In the case that $X$ is a differentiable manifold and the flow is differentiable and unstable this definition of separatrix coincides with that given by Markus in [4]. Roughly speaking the trajectories at which the orbit space fails to be Hausdorff are separatrices.

THEOREM 3.2. Let $\pi$ be a completely unstable flow on a locally compact, separable, metric space $X$. The set of separatrices is nowhere dense in $X$.

Proof. Let $S$ and $S_{1}$ denote the set of separatrices and the set of inseparable elements of $X / C$ respectively. Then $\bar{S}_{1}=S$. Set $F=\{U \subset X: U$ open and invariant, $U \neq X, J(U) \cap U=\varnothing$. It is easy to show that if $x$ is a 
wandering point, then there exists a neighborhood $V$ of $x$ such that $J(V) \cap V$ $=\varnothing$. Hence, $F$ covers $X$. Since $X$ is Lindelöf there is a countable family $\left\{U_{i}\right\}$ of elements of $F$ which cover $X$. Let $C_{1}$ be any element of $S_{1}$. Then there is a $C_{2} \in S_{1}$ such that $C_{1}$ and $C_{2}$ are inseparable. It is easy to show that $C_{1} \subset J\left(C_{2}\right)$. Let $j$ be such that $C_{2} \subset U_{j}$. Since $J\left(U_{j}\right) \cap U_{j}=\varnothing$ we must have $C_{1} \subset \partial U_{j}$. It follows that $S_{1} \subset \cup_{j=1}^{\infty} \partial U_{j}$. Since each $\partial U_{j}$ is nowhere dense, $S_{1}$ must be nowhere dense (Baire category theorem). Therefore $S$ is nowhere dense.

\section{REFERENCES}

1. J. Dugundji and H. Antosciewicz, Parallelizable flows and Liapunov's second method, Ann. of Math. (2) 73 (1961), 543-555. MR 23 \# A395.

2. O. Hajek, Dynamical systems in the plane, Academic Press, New York, 1968. MR 39 \# 1767.

3. K. Kuratowski, Topology, Vol. I, Academic Press, New York, 1968. MR 36 \#839.

4. L. Markus, Parallel dynamical systems, Topology 8 (1969), 47-57. MR 38 \#2806.

5. The global theory of ordinary differential equations, Lecture Notes, Univ. of Minnesota, 1964-1965.

6. R. McCann, Continuous flows with Hausdorff orbit spaces, Funkcial. Ekvac. 18 (1975), 195-206. MR 53 \# 11592.

7. V. V. Nemyckii, Topological problems of the theory of dynamical systems, Uspehi Mat. Nauk (N.S.) 4 (1949), no. 6 (34), 91-153; English transl., Amer. Math. Soc. Transl. (1) 5 (1962), 414-497. MR 11, 526.

Mathematics Department, Mississippi State University, Mississippi State, Mississippi 39762 